a key protective factor in improved well-being. Differences between clinical areas were also identified.

Conclusion. There was a clear increase in distress calls and requests to prescribe or increase psychotropic medication to calm the distress during the 'lockdown'. Changes in behavioural presentation may have occurred partly due to the disruption to the complex systems that typically support a child and the shift away from community support. Children with intellectual disability and their families are unique and embedded in complex systems comprising schools, respite, and healthcare provision which work together to deliver optimal mental healthcare with psychosocial interventions with medication for higher-risk situations. Any shifts in these systems may lead to an imbalance and a higher likelihood of medication use.

\section{Audit into post diagnostic support in newly diagnosed dementia patients}

Madhumanti Mitra* and Raghupathy Paranthaman

Greater Manchester Mental Health NHS Foundation Trust ${ }^{*}$ Corresponding author.

doi: 10.1192/bjo.2021.878

Aims. This audit aims to identify whether newly diagnosed dementia patients are offered post diagnostic support and potential factors influencing patient choice.

Background. A diagnosis of dementia can be life changing and hence post-diagnostic support for dementia is key. Multiple guidelines suggest that post diagnostic support need to be offered to all patients diagnosed with dementia. The Department of Health and Social Care and other national/ local guidelines suggest that post diagnostic support is offered to all patients diagnosed with dementia.

Method. Data were collected for 40 patients diagnosed with dementia. Using random number generator, patient group was selected from pool of patients diagnosed with dementia between July' 2017 - December' 2017. Data included whether they had been offered support during the initial appointment and what post-diagnostic support was offered. Demographic details obtained to identify patterns of support accessed by patients.

Result. All patients were offered post-diagnostic support. Diagnosis was discussed in appointment in about $93 \%$ of patients. Medication was discussed in $82 \%$ patients. Driving was discussed in only $64 \%$ patients and LPA was discussed in only $63 \%$ patients. When given choice between Post diagnostic support group (PDSG) and Dementia adviser (DA), slightly more women tend to choose PDSG group. The only 2 ethnic minority patients chose DA. $21 \%$ more patients opted for PDSG group when they had a carer. Conclusion. The positive is that some post-diagnostic support is offered to all patients. Although discussion of diagnosis with patients was done well, discussion of medication, driving and LPA can be improved upon. Ethnicity and family structure/ carer may have a bearing on patient choice of post-diagnostic support.

\section{Medication charts and consent to treatment documentation audit in an acute mixed in-patient psychiatry unit in south Manchester}

Madhumanti Mitra*, Shahid Hussain, Emma Raynor, Joanna Wong and Jennifer Thom

Greater Manchester Mental Health NHS Foundation Trust ${ }^{\star}$ Corresponding author.

doi: 10.1192/bjo.2021.879
Aims. The main aim of this audit was to look at documentation in medication charts in an acute mixed inpatient unit in South Manchester. In addition, we also looked at completion of capacity assessment and consent to treatment forms as appropriate.

Background. Safe prescription, administration and monitoring of medication is key to effective patient care. Due to the busy nature of inpatient hospital wards, errors do unfortunately occur both with the medications, and with the recording of their administration.

We will use a data collection tool to collect data as per standards described in our local GMMH policy. The medication chart will be used as the standard, as this is the current chart that is in use in the Trust.

Method. Data were collected from 31 medication charts for inpatients admitted in the ward between the $5 / 12 / 19$ to $18 / 12 / 19$. We captured data from each page of the medication chart that required a record to be made by any staff, including details of prescribing, administration and pharmacist checks. Data were recorded as either Yes/No or NA (Not Applicable). Data were then summarised and analysed using MS excel.

Result. Of the 31 patients, $22(71 \%)$ had a capacity assessment form completed and 16 (52\%) had a consent to treatment form completed. From the data analysis, it was clear that there are high rates of completion for the 'essential' parts of all prescriptions, including medicine name, dose, route and data. 'Route' was only recorded for $40 \%$ of prescriptions for depot medicines. Details of the administration of a medicine by a nurse was generally well-completed. For as required medications, all information relating to administration (date, time, dose and given by) were fully completed for $100 \%$ of prescriptions. For regular prescriptions however, the administration details were not as wellcompleted, where date of administration was recorded in $84 \%$ of prescriptions and signature in $29 \%$ of prescriptions. Unique patient identifiers are well-recorded on Page 1 of the prescription chart, though not maintained throughout the prescription chart. Nature of reaction to an allergy or sensitivity was only recorded in 6 of the 21 patients (29\%).

Conclusion. Overall, there were good completion rates for the mandatory parts of the prescriptions. However improvements could be made for prescriptions as well as administration and pharmacy checks. The capacity assessment and consent to treatment forms could be improved upon too. We plan to put the recommendations and re-audit in 3-6 months' time.

Service evaluation and research project: pros and cons of centralisation of ECT services in Pennine Care NHS Foundation Trust

Madhumanti Mitra* and Katherine Hayden

Pennine Care NHS Foundation Trust

${ }^{\star}$ Corresponding author.

doi: 10.1192/bjo.2021.880

Aims. The aim of this project would be to understand about the pros and cons/ benefits and risks of using a centralised model of ECT (currently being followed due to COVID restrictions) rather than a decentralised model of ECT (which has been the norm for a long time). A comparative account of both types of systems would help identify whether it could be implemented in Pennine Care and the results could be potentially transferable to other similar settings.

Method. An online survey was undertaken from staff members working in Pennine Care who have been involved in ECT delivery and management. The survey was undertaken between 07/12/ 
2020 and 11/01/2021 and included questions relating to preCOVID ECT (June 2019 to February 2020) and during-COVID ECT (March 2020 to November 2020). Questions were around travel of staff/patients for ECT, whether they had a preference for a model and their reasons around it. Data were summarised in MS Excel and free text comments analysed to gain an understanding of staff s preferences and reasoning behind their choices. Result. Although some boroughs had patients attending from other boroughs in pre-COVID times, considerably more staff (53.85\%) and patients (61.54\%) had to travel for ECT during COVID times. Around 50\% staff expressed a clear view for decentralised services; the common reasons being safer for patients, better continuity of care, less travelling issues, patients more likely to consent, easier to manage correct paperwork, easier to send staff who knows patients well, less driving for staff and likely less cancellations. Around $40 \%$ staff expressed a clear view for centralised services; the common reasons being less staff needed, better infection control, easier to maintain staff skills, efficiency, developing clinical expertise with larger number of cases, education opportunities, better set-up of clinics, transportation and accessibility. Some concerns raised for the centralised model were managing patients with complex anaesthesia, travelling for unwell/ disturbed patients, too high patient numbers, poor communication and impact on training.

Conclusion. In summary, there was a mixed view of which services are preferable. Further discussion in trust wide ECT forum will be helpful to move things forward. Although it is likely that services may shift from a decentralised to a centralised system, we need to ensure this is done safely and in particular, address the main concerns around centralisation.

\section{Preliminary investigation into the identification and management of catatonia in patients admitted to adult inpatient units}

Joanna Moore ${ }^{1 \star}$, Amy Kunicki², Georgina Latcham², Eleanor Perkins ${ }^{2}$ and Emma Vaccari ${ }^{2}$

${ }^{1}$ Dorset Healthcare University NHS Foundation Trust and ${ }^{2}$ Southern Health NHS Foundation Trust

${ }^{*}$ Corresponding author.

doi: 10.1192/bjo.2021.881

Aims. The prevalence of catatonia is considered to be approximately $10 \%$ in psychiatric inpatients. Clinical experience suggests a lower documented prevalence. This could cause longer admissions and complications, such as Neuroleptic Malignant Syndrome (NMS). We carried out a service evaluation to investigate the recognition and management of catatonia on inpatient units in Southern Health Foundation Trust (SHFT). We reviewed the local documented prevalence of catatonia, treatment offered and prevalence of complications.

Method. We retrospectively reviewed the electronic records of 95 consecutive admissions to four adult inpatient units in SHFT, starting on 1st August 2020. We reviewed notes for the admission to establish whether catatonia was suspected and identified. We applied the screening questions from the Bush-Francis Catatonia Rating Scale (BFCRS) to the documented mental state examinations (MSE) prior to, and shortly after, admission. We also recorded the prescriptions issued during the first 72 hours of admission, and whether patients developed neuroleptic malignant syndrome (NMS), serotonin syndrome or required admission to a general hospital during admission.

Result. Catatonia was documented as a possibility for 2 patients (2.1\%). One showed possible posturing and stupor, while there were no documented symptoms for the other. In both cases the possibility was discounted by the clinical team. Twelve patients (12.6\%) showed one or more possible or confirmed signs of catatonia. Eleven of these were prescribed regular antipsychotic medication on admission, but only 3 were prescribed regular benzodiazepines. NMS was more likely to be suspected in patients with a BFCRS of 1 or more compared with those with a score of 0 , with an odds ratio of 8.1 (95\% CI [1.03-64.0], Fisher's exact test = $7.79, \mathrm{p}=.076$ ).

Conclusion. Catatonia is likely under-recognised and undertreated locally among psychiatric inpatients. Although only approaching statistical significance, the higher rate of suspected NMS in patients showing possible catatonia is noteworthy and needs further investigation. Regular benzodiazepines were not frequently prescribed in this group, while antipsychotics, prescribed in all of these patients, can precipitate NMS. Alternatively, this finding could reflect the overlap in clinical presentation between NMS and catatonia. Data collection was limited by the frequent use of "remote clerking", in the context of the COVID-19 pandemic. Additionally, the quality of mental state examinations was often not sufficient to draw any conclusions on the possible presence or absence of catatonic symptoms. This project has highlighted practice in need of improvement, which will be further prospectively investigated and improved via a Quality Improvement Project.

\section{Physical health monitoring of patients prescribed depot antipsychotic medication in north west Edinburgh community mental health team}

Douglas Murdie $^{1 \star}$, Jakub Wojtowicz ${ }^{2}$, Alexandra Thompson ${ }^{2}$, Anne MacLeod ${ }^{2}$, Adam Mallis ${ }^{2}$, Hamsi Evans², Joshua Haggart ${ }^{2}$, Hae Choi $^{2}$ and Vikki Argent ${ }^{3}$

${ }^{1}$ Consultant Psychiatrist, NW CMHT, NHS Lothian; ${ }^{2}$ University of Edinburgh Medical School - 1st year Medical Student and ${ }^{3}$ Consultant Perinatal Psychiatrist, Perinatal MHT, NHS Lothian ${ }^{\star}$ Corresponding author.

doi: 10.1192/bjo.2021.882

Aims. To monitor the quality of physical health monitoring of patients prescribed depot antipsychotic medication in the North West Edinburgh Community Mental Health Team (CMHT). We also evaluated the completeness of prescriptions and Mental Health Act (Scotland) (Act) 2003 paperwork where relevant.

Background. Antipsychotic medications are medicines for treating conditions such as Schizophrenia, but some may be associated with an increased risk of Metabolic Syndrome. Moreover, evidence indicates that patients with major mental disorder have a reduced life expectancy in comparison to those without such diagnoses. These two factors illustrate the importance of the physical health of this patient cohort being monitored on a regular basis. This project will evaluate how a local CMHT is performing, with the possibility of enacting service improvements if required.

Method. The records of the 60 patients prescribed depot antipsychotic medication administered by this CMHT were reviewed. A check-list was created consisting of 14 categories analysing the quality of physical health monitoring, as well as compliance with prescription standards and, where relevant, Mental Health Act (Scotland) (Act) 2003 paperwork. We compared patient records against our checklist for the calendar year of 2019. The Scottish Intercollegiate Guidelines Network (SIGN) 131 (Management of Schizophrenia) section 5.2 was used as the gold standard for physical health monitoring against which the data we collected was compared. 